\title{
Experimental Comparison of Type-1 and Type-2 Fuzzy Logic Controllers for the Control of Level and Temperature in a Vessel
}

\author{
B. Cosenza, M. Galluzzo \\ Dipartimento di Ingegneria Industriale, Università degli Studi di Palermo, Viale delle \\ Scienze, Ed. 6, 90128 Palermo, Italy
}

\begin{abstract}
The objective of this experimental study is to compare the performance of type- 1 and type-2 fuzzy logic controllers on a real system where the control of liquid level and temperature are considered. By the use of genetic algorithms it is possible to optimize the fuzzy sets of each fuzzy controller assuring high control performance. The experimental results show that a better control in terms of robustness can be achieved by type-2 fuzzy logic controllers.
\end{abstract}

Keywords: type-1 fuzzy logic controller, type-2 fuzzy logic controller, genetic algorithms.

\section{Introduction}

It is well known that it is not possible to obtain good performances with traditional controllers when the processes to be controlled are characterized by high nonlinearity or uncertainties. Therefore many nonlinear controllers like fuzzy logic controllers have been reported to be successfully used just for their robustness and for their ability to handle the system nonlinearities. Fuzzy logic controllers are usually built up using type1 fuzzy sets and are referred as type-1 FLCs (Mandani, 1974). Recently it has been shown that a new kind of fuzzy controllers, the "type-2" FLCs (Karnik and Mendel, 1998), can better handle all nonlinearities and uncertainties present in a system, making use of particular fuzzy sets, defined as "type-2 fuzzy sets". In many control fields as bioprocess control (Galluzzo and Cosenza, 2010), control of autonomous mobile robots (Martinez et al., 2009), anesthesia control (Castillo et al., 2005), control for quarter vehicle active suspensions (Cao et al, 2008), level control (Wu and Tan, 2006), the superiority of type-2 FLCs over their type-1 counter-parts has been successfully shown and confirmed.

In spite of their superiority there are not many applications of type-2 fuzzy logic controllers to real systems. The main aim of this paper is therefore to test on a laboratory experimental system their concrete applicability and to compare their performance with type-1 fuzzy logic controller performance.

Type-2 FLCs give better results than their type-1 counterparts above all in environments full of uncertainties. The main characteristic of type-2 fuzzy sets is just their ability to handle uncertainties more efficiently than type-1 FLCs. This is possible because a larger number of parameters and more freedom degrees are available with type-2 fuzzy sets. 
In this work the design of type-2 FLCs is carried out optimizing the controller fuzzy sets by a technique that uses genetic algorithms. Also type-1 FLCs used for comparison are optimized with the same technique. Type- 1 and type-2 FLCs are tested in a real system for the control of temperature and liquid level in a vessel. In the experimental system uncertainties are present or have been introduced as measurement noises.

\section{Experimental rig}

The experimental system consists of a simple cylindrical pressurized vessel in which water is heated by an electrical coil. The flow rate of the water leaving the system is proportional to the square root of the height of the water in the tank. This term constitutes the main source of nonlinearity in the system. Additional non linearities and uncertainties are present as variable transport delay and noise in the sensor outputs.

\section{Interval type-2 fuzzy logic}

An interval type-2 fuzzy set $\tilde{A}_{I}$ is defined as follows:

$$
\tilde{A}_{I}=\int_{x \in X} \int_{u \in J_{x} \subseteq[0,1]} 1 /(x, u)
$$

Therefore the secondary grade of interval set belongs to interval $[0,1]$.

The main characteristic of type- 2 fuzzy sets is their ability to take in to account the uncertainty of a system. This is possible through a bounded region (Fig. 1a) in the membership functions that is called Footprint of Uncertainty (FOU). The FOU can be described in terms of upper (UMF) and lower (LMF) membership functions. In the real study case the measurement noise is the main source of uncertainty and by use of the FOU it is possible to capture the uncertainty, minimizing consecutively its negative effects on the control system.

(a)

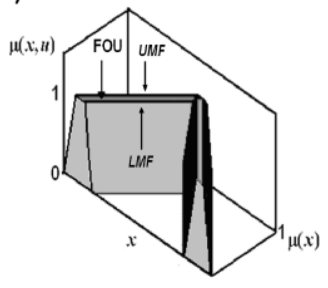

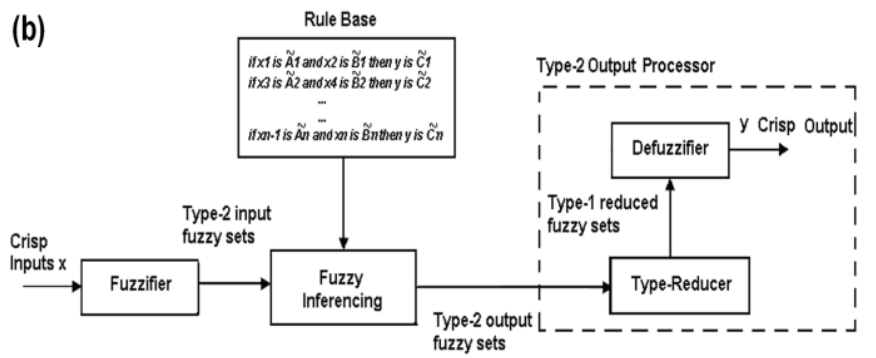

(b)

Fig. 1. a) Three-dimension Interval Type-2 Triangular Fuzzy Set. b) FOU in terms of Upper and Lower Membership Functions.

As type-1 fuzzy logic systems, type-2 fuzzy logic systems contain four components as well: a rule-base, a fuzzifier, an inference-engine and an output-processor. The last component (the output-processor) is just the main difference between type- 1 and type- 2 FLS. It maps a type-2 fuzzy set into a type-1 fuzzy set and then transforms (as a normal type-1 defuzzifier) the fuzzy output in a crisp output (Fig. 1b). 
Experimental comparison of type-1 and type-2 fuzzy logic ocntrollers for the control of level and temperature in a vessel

\section{Controller optimization by genetic algorithms}

The parameters of type-1 and type- 2 fuzzy controllers for the control of level and temperature were optimized with genetic algorithms. Genetic algorithms are an optimization technique that discovers more than one solution to a problem (Holland, 1975). The approach for selecting the parameters of a type-2 fuzzy logic system is the totally independent approach. In this optimization method all the parameters of the type-2 fuzzy logic systems are tuned, without the aid, as reference, of a type-1 fuzzy logic system. The method avoids local minima and assures great design flexibility.

The parameter optimization process of fuzzy controllers with genetic algorithms is based on the simulation of the system controlled by the fuzzy controllers being designed. Because of the approximation that characterizes the system model, the performance of the designed controllers may deteriorate when they are used in the real system. Therefore each fuzzy controller is exposed to model uncertainties during the phase of controller design ( $\mathrm{Wu}$ and Tan, 2006), to preserve in the real application, the good results obtained in simulation. To create the effect "uncertainty" four different plant models were used in simulation and different plant conditions were considered for each model.

The same genetic algorithms are used to evolve the sets of type- 1 and type- 2 fuzzy controller parameters. They make use of the sum of the integral of the time weighted absolute error (ITAE) obtained from the four plant models, to evaluate the fitness function of each candidate solution.

\section{Experimental results}

Some experimental results are shown in the following figures. The response of the level to a change in the set-point value from 0 to $4 \mathrm{~cm}$ at instant $\mathrm{t}=10 \mathrm{sec}$ and from 4 to $6 \mathrm{~cm}$ at about $\mathrm{t}=100 \mathrm{sec}$ is reported in Fig. 2. In these conditions there is a very slight difference between the performance of type- 1 and type-2 FLCs. The difference becomes more evident introducing a noise in the level measurement (Fig. 3 a,b).

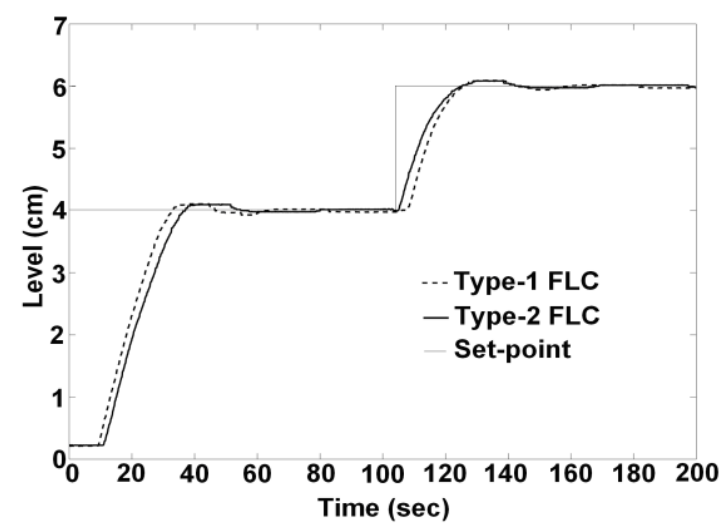

Fig. 2. Type-1 FLC vs. Type-2 FLC. a) Response to a level set-point change (from 0 to 4 at about $\mathrm{t}=10 \mathrm{sec}$ and from 4 to 6 at about $\mathrm{t}=100 \mathrm{sec}$. 
a)

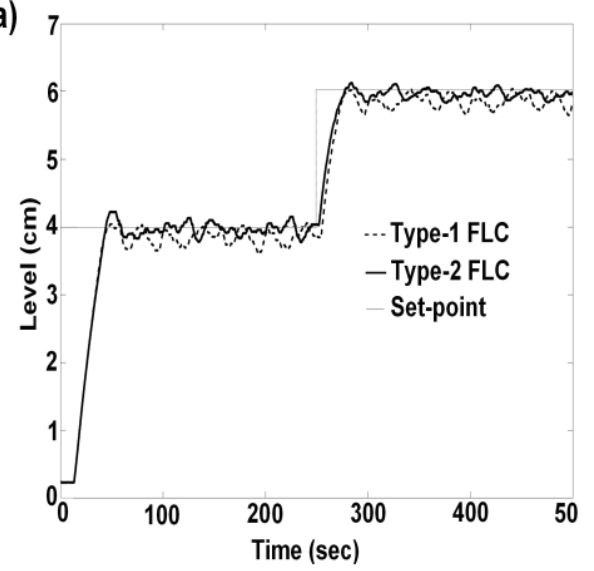

b) 7

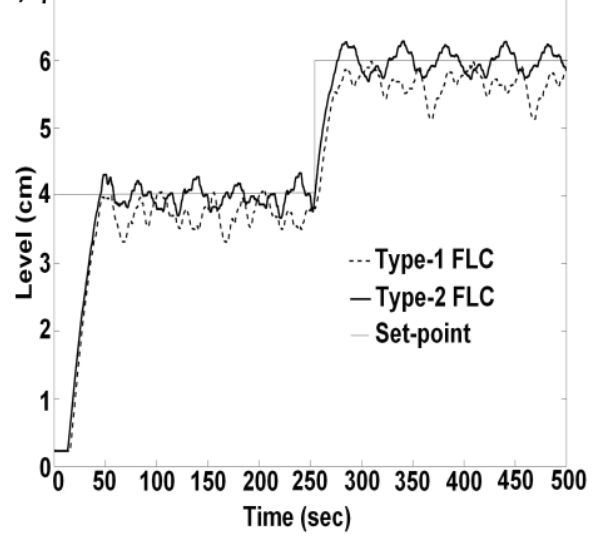

Fig. 3 a) Response to a level set-point change (from 0 to 4 at about $\mathrm{t}=10 \mathrm{sec}$ and from 4 to 6 at about $\mathrm{t}=250 \mathrm{sec}$ ) with a small amplitude of noise. b) Response to a level setpoint change (from 0 to 4 at about $t=10 \mathrm{sec}$ and from 4 to 6 at about $t=250 \mathrm{sec}$ ) with a larger amplitude of noise.

In Fig. 3a the behavior of the system controlled by type-2 FLC is characterized by oscillations with a smaller amplitude value than that of type-1 FLC; moreover an offset is present only in the answer of the system controlled by type-1 FLC. The result shown in Fig. 3b was obtained artificially increasing the noise amplitude in the level measurement. It confirms the previous result, with type-1 FLC showing the worst performance and increasing the off-set for both set-point changes.

A change in the set-point value from 29 to $30^{\circ} \mathrm{C}$ at $\mathrm{t}=0 \mathrm{sec}$ was considered as shown in Fig. 4. Also for the temperature control, the performance of the type-2 FLC is better than its type- 1 counterpart, with the difference that in this case the addition of an artificial noise was not necessary because the temperature measurement is already characterized by a large noise.

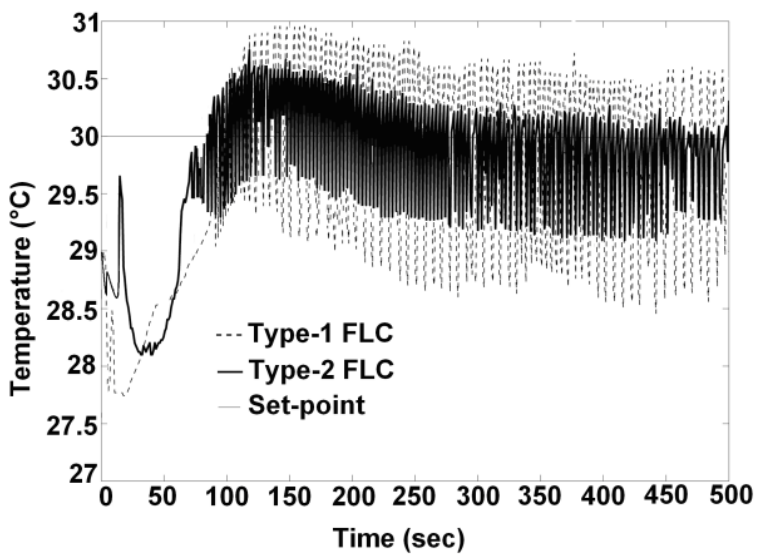

Fig. 4 Response to a temperature set-point change (from 29 to 30 at $\mathrm{t}=0 \mathrm{sec}$ ).

The temperature oscillates in both cases around the set-point value but the type-2 FLC is able to decrease the oscillation amplitude more than the type-1 FLC. 
Experimental comparison of type-1 and type-2 fuzzy logic ocntrollers for the control of level and temperature in a vessel

\section{Conclusions}

Type-2 FLCs are able to control the temperature and level in the system more efficiently than type-1 FLCs especially when uncertainties due for instance to measurement noises are present.

Both type-1 and type-2 fuzzy controllers used in the study were optimized by a genetic algorithm method based on the totally independent approach.

Increasing the uncertainty degree of the control system, the difference between the performance of type-1 and type-2 FLCs becomes more evident.

Type-2 FLCs represent an effective solution for control problems originated by parameter uncertainty and measurement noise.

\section{Acknowledgments}

Authors are grateful to Dr. D. Wu and Dr. W. W. Tan for making available their genetic algorithm code.

\section{References}

Cao, J., Liu, H., Li, P., Brown, D., 2008. Adaptive Fuzzy Logic Controller for Vehicle Active Suspension with Interval Type-2 Fuzzy Membership Functions, in Proc. FUZZ-IEEE 2008, Hong Kong, 1361-1373.

Castillo, O., Huesca, G., Valdez, F., 2005. Evolutionary Computing for Optimizing type-2 Fuzzy Systems, Intelligent Control of Non-Linear Dynamic Plants, in: Proc. North American Fuzzy Info, Processing Society (NAFIPS 2005), Ann Arbor, MI, 6, 247-251.

Galluzzo, M., Cosenza, B., 2010. Adaptive Type-2 Fuzzy Logic Control of a Bioreactor, Chemical Engineering Science, 65 (14), 4208-4221

Holland, J.H., 1975. Adaptation in natural and artificial systems, University of Michigan Press, Mi.

Karnik, N.N., Mendel, J.M., 1998. Introduction to type-2 fuzzy logic systems, in Proc. $7^{\text {th }}$ Intl. Conf. on Fuzzy Systems FUZZ- IEEE 1998, Anchorage, AK, 915-920.

Mamdani, E.H., 1974. Application of fuzzy algorithms for control of simple dynamic plants, in Proc. of IEEE, 121 (12), 1585-1588.

Martinez, R., Castillo, O., Aguilar, L.T., 2009. Optimization of interval type-2 fuzzy logic controllers for a perturbed autonomous wheeled mobile robot using genetic algorithms, Journal of Information Sciences, 179 (13), 2158-2174.

Wu, D., Tan, W.W., 2006. Genetic learning and performance evaluation of interval type-2 fuzzy logic controllers, Engineering Applications of Artificial Intelligence, 19, 829-841. 